\title{
Implantação de uma Liga Acadêmica de Anatomia: Desafios e Conquistas
}

\author{
Implementation of an Academic League of \\ Anatomy: Challenges and Achievements
}

Jorge Henrique Santos da Silva

Larissa Grasieli Chiochettal

Luana Fraga Torres de Oliveiral

Vivian de Oliveira Sousa ${ }^{I}$

PALAVRAS-CHAVE

- Anatomia;

- Universidades;

- Ensino;

- Pesquisa;

- Educação Médica.

\section{KEYWORDS}

- Anatomy;

- Universities;

- Education;

- Research;

- Medical Education.

Recebido em: 09/12/2014

Aprovado em: 30/03/2015

\section{RESUMO}

As Ligas Acadêmicas exercem papel fundamental nas universidades, com atividades extracurriculares que expandem o conhecimento dos alunos integrantes além da graduação, contemplando também o meio acadêmico e a sociedade. O processo pioneiro de implantar uma Liga Acadêmica em um campus em implantação é um grande desafio. A Liga Acadêmica de Anatomia do Campus UFRJ-Macaé (Laanamac) objetiva estimular a interação dos alunos, desenvolver projetos de pesquisa, extensão $e$ ensino, e ser modelo para a criação de novas ligas. Este trabalho relata o primeiro ano da Laanamac e os resultados alcançados: maior interesse dos alunos pela Anatomia, possibilidade de ingresso em uma Iniciação científica, dissecação de cadáveres, interação com docentes e discentes de outras instituições, desenvolvimento de habilidades como gerenciamento de uma liga e organização de eventos, $e$ divulgação do nome da instituição em congressos. Uma liga recém-formada pode contribuir de forma significativa no desenvolvimento de novos campi, por ampliar as possibilidades, principalmente, dos que estão se estabelecendo longe das grandes cidades.

\begin{abstract}
The Academic Leagues play a fundamental role in universities, offering extracurricular activities that expand students' knowledge beyond undergraduate learning, including academia and society. However, the pioneering process of implementing an Academic League at a campus under construction is a great challenge. The Academic League Anatomy of the UFRJ Macaé Campus (Laanamac) aims to encourage student interaction, develop research, community outreach and teaching projects and to be a model for the creation of new leagues. This paper reports on the first year of Laanamac and its achievements, such as increased student interest in anatomy, the opportunity to partake in a Scientific Initiation, the dissection of corpses, interaction with teachers and students from other institutions, the development of skills such as league management and organization of events, and the promotion of the institution's name at congresses. A newly formed league can add significantly to the development of new campuses, by opening up new possibilities, especially for those who are establishing themselves far from the big cities.
\end{abstract}




\section{INTRODUÇÃO}

As Ligas Acadêmicas (LA) são organizações estudantis, com supervisão de docentes ou profissionais vinculados a uma instituição, de duração indeterminada, sem fins lucrativos, que visam aprimorar o estudo e habilidades adquiridas na graduação de uma área específica do conhecimento, visando integrar acadêmicos de diversos períodos e cursos que tenham interesse nessa área ${ }^{1}$. A participação em ligas é uma atividade extracurricular frequentemente desenvolvida por acadêmicos de Medicina ${ }^{2}$.

Segundo as discussões na literatura e o cenário socioeconômico e cultural do País, pressupõe-se que as finalidades da educação superior não se limitem apenas à formação acadêmica, mas envolvam um conjunto de medidas intencionais e subjetivas que tornam a formação profissional mais holística e abrangente, mantendo a interação entre a academia e a população, enfatizando o compromisso da universidade com a cidadania, além das ações educativas encontradas em sua estrutura curricular².

Essa forma de aperfeiçoar e praticar o aprendizado, além do adquirido na graduação, surgiu no Brasil em 1920 com a criação da Liga de Combate à Sífilis, na Faculdade de Medicina da Universidade de São Paulo, na qual os estudantes integraram seus conhecimentos acadêmicos e construíram postos para cuidar de pacientes acometidos por essa afecção, aprimorando o estudo sobre a sífilis e melhorando a qualidade de vida das pessoas que os procuravam ${ }^{3}$. Porém, o movimento crescente de criação das LA se iniciou no período da Ditadura Militar, quando o questionamento do ensino universitário e a aplicabilidade dos avanços técnico-científicos foram mais intensos. A partir da Constituição de 1988, em que foi elaborado o princípio da indissociabilidade entre ensino, pesquisa e extensão ${ }^{4}$, as LA ganharam maior atuação nas universidades, abordando diferentes áreas médicas, mas adotando o mesmo caráter social, acadêmico e científico da sua instituição de origem. No final da década de 1990, várias discussões sobre a educação médica abordaram o incremento das LA nas grades curriculares das escolas médicas, tornando o papel das LA ainda mais relevante ${ }^{5}$. Desde então, as LA se fortaleceram e contribuíram ainda mais com a graduação e a população. Em setembro de 2006, no $8^{\text {o }}$ Congresso Brasileiro de Clínica Médica, foi criada a Associação Brasileira das Ligas Acadêmicas de Medicina (Ablam), fundamentando a padronização das LA nos diversos cenários nacionais, sendo um marco na história da medicina brasileira ${ }^{5}$.

A Faculdade de Medicina do Campus Macaé da Universidade Federal do Rio de Janeiro (Campus UFRJ-Macaé), criada em 2007 através da interiorização da universidade, recebeu os primeiros acadêmicos em agosto de 2009. Em novembro de 2013, a coordenação do curso incentivou os alunos a organizarem LA em diversas áreas, dentre elas a de Anatomia. Dessa forma, começou um processo de recrutamento dos estudantes que portavam interesse nessa área. Inicialmente, um grupo de 21 alunos distribuídos do segundo ao oitavo período foi reunido. A partir de então, estes se organizaram para fundar a Liga Acadêmica de Anatomia do Campus UFRJ-Macaé (Laanamac) com o intuito de acrescentar à graduação o conhecimento mais minucioso repleto de experiência dos acadêmicos sobre a Anatomia, deixando-a mais dinâmica para os alunos. Este trabalho relata a experiência de implantar uma LA em um campus novo no interior e mostra como isto contribuiu para o desenvolvimento dos alunos de Medicina e, consequentemente, para o Campus UFRJ-Macaé.

\section{RELATO DE EXPERIÊNCIA}

A Liga Acadêmica de Anatomia do Campus UFRJ-Macaé foi fundada em 4 de fevereiro de 2014 por um grupo de 21 alunos de Medicina distribuídos do segundo ao oitavo período da graduação que buscavam, entre outros objetivos, aprimorar seus conhecimentos sobre a Anatomia. Este processo mostrou as dificuldades de implantação de uma LA de Medicina numa universidade, e o pioneirismo da Laanamac tornou-se seu primeiro desafio, uma vez que não havia nenhuma LA no campus como modelo para auxiliar neste início. Nesse momento, foi fundamental a consulta à Ablam por meio de suas diretrizes e a busca por outras ligas da mesma área de interesse para compartilhar informações sobre a construção e funcionamento da LA.

Inicialmente, a diretoria da Laanamac foi composta por seis cargos (presidente, vice-presidente, secretário-geral, diretor Administrativo/Financeiro, diretor Científico/Extensão e diretor de Comunicação). A confecção do estatuto foi baseada em outros estatutos de LA de Anatomia. A Laanamac manteve seu regimento com base nas diretrizes da Ablam para, além de estar apta a associar-se, ter um modelo de normatização de uma LA, evitando que a Laanamac fosse apenas um grupo de estudantes sem supervisão com demandas pessoais afins que se especializavam em Anatomia 6 .

Para a organização inicial, os membros se reuniam semanalmente para discutir sobre a implantação da liga e as atividades que deveriam ser realizadas. Desta forma, surgiram as aulas de Anatomia construídas pelos membros da Laanamac com os mesmos temas da graduação, porém com ênfase nos dados mais utilizados na prática clínica/cirúrgica, preparando os ouvintes para o cotidiano médico vivenciado pelos acadêmicos. Estas aulas teóricas e práticas trouxeram temas interessantes e diferentes formas do estudo de Anatomia. 
Além das aulas ministradas pelos membros da liga, foi organizada uma aula inaugural da Laanamac, ministrada por um médico, com o objetivo de apresentar a liga ao corpo social da Universidade (Figura 1). Esta palestra sobre Medicina do Esporte e Anatomia reuniu alunos, professores e coordenadores de cursos da área de saúde, gerando um diálogo multidisciplinar sobre um tema que está em nossas vivências e trazendo uma visão diferencial sobre a utilização da Anatomia em diversas situações, sejam profissionais ou de lazer.

Figura 1

Material de divulgação da aula inaugural da Liga Acadêmica de Anatomia do Campus UFRJ-Macaé

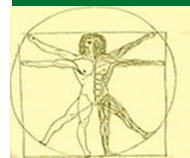

LIGA ACADÊMICA DE ANATOMIA DA UFRJ - CAMPUS MACAÉ (LAANAMAC)

Convida todos os estudantes da área da saúde para sua aula inaugural sobre:

\section{MEDICINA DO ESPORTE}

Ministrada pel@ Professor Roberto Anion -Médico do Esporte e Ortopedista-

\section{Dวْอะ 21 de Maio}

กำละ 18 horas

டంธaी: Cidade Universitária - Bloco B . Sala 301/302

-VAGAS LIMITADAS- Os interessados em participar deverào procurar os membros da LAANAMAC e realizar a inscriçăo cujo valor será de R\$3,00. AO FINAL HAVERÁ COFFEE BREAXI!

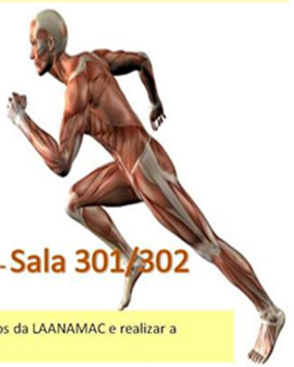

A busca por outras ligas de Anatomia que auxiliassem a implantação da liga no Campus UFRJ-Macaé proporcionou maior interação com os alunos da sede da UFRJ. A Liga de Anatomia da UFRJ (Laanato) convidou alguns membros da liga do Campus UFRJ-Macaé a participarem de uma aula de Anatomia Palpatória ministrada no Instituto de Ciências Biomédicas da UFRJ. Esta aula proporcionou o conhecimento dos acidentes ósseos de maneira dinâmica. Posteriormente, os participantes replicaram o conhecimento para todos os membros da Laanamac.

A Laanamac propôs ainda aos seus membros atividades práticas, como dissecção de cadáveres e oficinas de suturas. O conhecimento profundo da Anatomia é pré-requisito para disciplinas do ensino médico. Ao realizar inspeção, palpação, percussão, ausculta e análise de exames de imagem, torna-se necessário o conhecimento da localização dos órgãos e particularidades das estruturas anatômicas. Desta forma, as atividades práticas relacionadas à Anatomia desenvolvem e preparam melhor o acadêmico para a prática médica.
Em seu primeiro ano de existência, a Laanamac organizou, em parceria com docentes e com a Laanato (liga de Anatomia da sede), o I Simpósio de Anatomia do Campus UFRJ-Macaé (Figura 2). O simpósio ocorreu no dia 25 de agosto de 2014 no Campus UFRJ-Macaé e contou com professores e técnicos de Anatomia da UFRJ e de outras instituições, com a realização de um workshop em Anatomia Palpatória e com a presença da presidente da Sociedade Brasileira de Anatomia à época.

\section{FIgURA 2}

I Simpósio de Anatomia do Campus UFRJ-Macaé

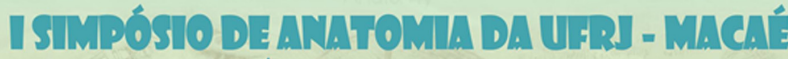 CIISTMÓSO DE ANATOMTLA. URT}

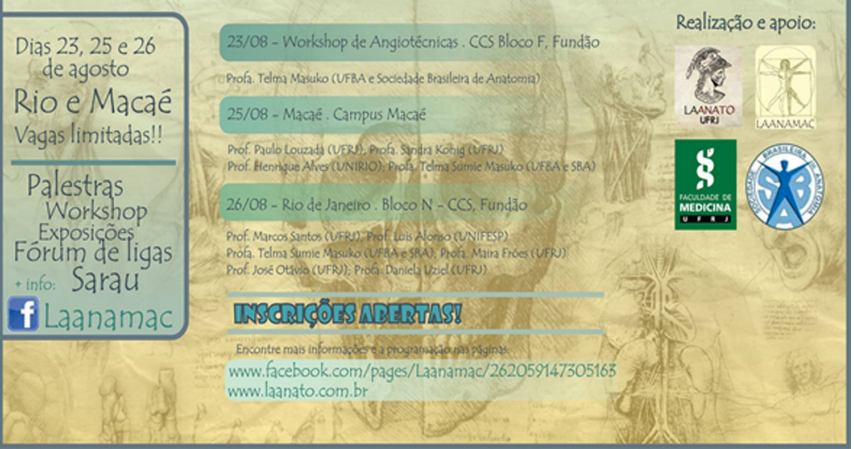

A base das LA no tripé ensino-pesquisa-extensão despertou maior interesse dos acadêmicos de Medicina por projetos de pesquisa e extensão. Os membros da Laanamac produziram trabalhos relacionados ao ensino da Anatomia no Campus UFRJ-Macaé apresentados em congressos nacionais e na Jornada de Pesquisa e Extensão da universidade.

\section{RESULTADOS E DISCUSSÃO}

O estabelecimento da Laanamac resultou em maior comprometimento dos alunos do Campus UFRJ-Macaé com o ensino de graduação, aprofundamento dos conhecimentos de Anatomia e maior aplicabilidade dos conteúdos, inserção dos alunos em atividades de pesquisa e extensão, e interação com alunos de outras instituições.

A interação entre alunos de diferentes cursos e períodos do Campus UFRJ-Macaé promoveu uma troca de experiências e valores que dificilmente poderia ocorrer no cotidiano de grades cheias de atividades acadêmicas diferentes. Sabe-se da importância de uma equipe interdisciplinar na saúde para oferecer um tratamento de qualidade. Todavia, a interdisciplinaridade necessita do estabelecimento de vínculos no processo de trabalho ${ }^{7}$. Esta integração nem sempre é facilmente concebida. 
A troca de saberes dentro de uma disciplina comum a diferentes cursos já no início de uma graduação pode ter resultados benéficos na facilitação dos vínculos necessários a uma efetiva equipe interdisciplinar após a formação destes alunos.

A Laanamac propôs atividades como aulas formuladas pelos próprios membros com a visão do acadêmico no cenário prático de sua profissão, resgatando a importância dos temas abordados na graduação, tendo em vista que a participação em atividades extracurriculares não se baseia apenas em preencher lacunas curriculares, mas também na integração, com troca de experiências entre os colegas, atendendo às indagações profissionais ${ }^{8}$. Além disso, com a reformulação das grades curriculares das escolas médicas com enfoque nas necessidades de saúde da população e do Sistema Único de Saúde ${ }^{9}$, a carga horária das disciplinas do ciclo básico está cada vez mais reduzida, dificultando o desenvolvimento de atividades que poderiam dar mais aplicabilidade aos conteúdos ministrados. É importante ressaltar que a Laanamac não objetiva suplementar um ensino deficiente de Anatomia. Currículos com falhas em áreas relevantes à formação médica devem ser corrigidos e não complementados por LA ${ }^{10}$. As aulas da Laanamac são abertas ao público sem distinção de conhecimento prévio do assunto, a fim de minimizar qualquer limitação à participação de pessoas que, mesmo não tendo Anatomia em sua grade curricular, se interessem pelo assunto.

As atividades de ensino desenvolvidas pela liga incentivam o magistério superior. De forma geral, este incentivo é comum nas universidades por meio da monitoria acadêmica ${ }^{11,12}$. O monitor comumente tem oportunidades de ministrar aulas práticas supervisionadas, principalmente nesta cadeira específica, Anatomia. Esta atividade apresenta benefícios gerais, como a oportunidade de o estudante desenvolver habilidades inerentes à docência, aprofundar conhecimentos na área específica e contribuir com o processo de ensino-aprendizagem dos alunos monitorados ${ }^{13}$. De modo semelhante, a responsabilidade de organizar aulas de Anatomia com ênfase médica pelos membros da Laanamac alicerça melhor o conhecimento nesta área específica de estudo e potencializa o senso crítico acadêmico e profissional, tanto para seguir a carreira docente quanto para servir a sociedade em geral. Ramalho e colaboradores avaliaram o aprendizado dos integrantes de uma LA de Anestesiologia após um ano de participação em suas atividades e concluíram que a liga é um instrumento útil de ensino e promove ganho de aprendizado ${ }^{14}$.

As atividades de ensino são comuns nas LA, todavia as atividades de pesquisa e extensão não são bem desenvolvidas ${ }^{10}$. Para evitar este erro, desde sua fundação, a Laanamac planejou projetos de pesquisa e extensão com o objetivo de abordar a comunidade e o meio acadêmico, tomando cuidado para evitar que a Laanamac também se transforme em atividades de iniciação científica e assistência, sem a função primordial de extensão universitária, prevenção de doenças e promoção da saúde ${ }^{2}$, e se torne um grupo de estudantes com demandas pessoais que visem apenas aprofundar um assunto específico, como se fosse uma especialização precoce ${ }^{15}$. A especialização precoce favorecida pela participação em LA tem sido discutida. O relato de uma Liga de Cirurgia Plástica revelou que $28,6 \%$ dos alunos tinham interesse em atuar em Cirurgia Plástica quando ingressavam na liga. Após um ano de participação, este número subiu para 78,6\% 6 . Discute-se se o fato de participar de uma LA não deve restringir as visões de possibilidades dos estudantes de Medicina. No caso da Anatomia, uma disciplina transversal a diversas especialidades médicas, este viés é minimizado.

As atividades de pesquisa são de grande importância para a formação médica, pois favorecem a capacidade crítica e maturidade científica, e, de modo geral, o ambiente das LA tem estimulado os alunos a desenvolver projetos de iniciação científica $^{5}$. Mas ainda não são todas as que apresentam trabalhos em congressos, e poucas publicam em periódicos, pois pouco do que é produzido se torna divulgação científica ${ }^{10}$. Já no primeiro ano da Laanamac, alguns alunos se envolveram em projetos de iniciação científica, e oito membros tiveram trabalhos aceitos em congressos importantes no cenário nacional, como o Congresso Brasileiro de Educação Médica, Congresso Brasileiro de Anatomia e Jornada de Pesquisa e Extensão da UFRJ. Isto mostra que o incentivo inicial às atividades de pesquisa pode resultar em frutos de divulgação científica (Quadro 1).

Ainda assim, corre-se o risco de a LA se transformar em sociedades científicas, desconsiderando a extensão universitária e seu papel transformador da sociedade. A Laanamac, desde seu início, planejou atividades de extensão com auxílio e orientação de professores de Anatomia. Os projetos iniciais envolvem a integração entre ensino fundamental e médio com o ensino superior, levando os saberes acadêmicos gerados na universidade e um estímulo às ciências da saúde aos alunos da rede pública de ensino da cidade onde o campus está inserido.

Além do desenvolvimento das atividades de ensino, pesquisa e extensão, a interação dos membros da Laanamac com as outras ligas de Anatomia proporcionou grande troca de experiências e ampliação do conhecimento. A primeira parceria foi com a Liga de Anatomia da sede da instituição, a Laanato, que sempre promoveu o ensino da Anatomia, multiplicando os saberes para outras instituições. Os encontros com outras LA propiciaram a formação de um grupo das Ligas de Ana- 
QuAdro 1

Trabalhos desenvolvidos pelos membros da Laanamac apresentados em congressos e jornadas nacionais

\begin{tabular}{|l|l|}
\hline \multicolumn{1}{|c|}{ Título dos trabalhos aceitos } & \multicolumn{1}{c|}{ Congresso } \\
\hline $\begin{array}{l}\text { Criação e implantação da Liga Acadêmica de Anatomia } \\
\text { da UFRJ Campus Macaé: desafios, objetivos e resultados }\end{array}$ & $\begin{array}{l}\text { 1. XXVI Congresso Brasileiro de Anatomia (CBA) e II Encontro de Ligas Estudantis } \\
\text { de Morfologia }\end{array}$ \\
$\begin{array}{l}\text { 2. Jornada de Pesquisa e Extensão da UFRJ (JPE) } \\
\text { 3. Congresso Brasileiro de Educação Médica (Cobem) }\end{array}$ \\
$\begin{array}{l}\text { Imaginologia no ensino da Anatomia Humana: } \\
\text { perspectiva dos alunos de Medicina do Campus UFRJ- } \\
\text { Macaé }\end{array}$ & $\begin{array}{l}\text { 1. XXVI Congresso Brasileiro de Anatomia (CBA) e II Encontro de Ligas Estudantis } \\
\text { de Morfologia } \\
\text { 2. Jornada de Pesquisa e Extensão da UFRJ (JPE) }\end{array}$ \\
\hline $\begin{array}{l}\text { Metodologias ativas e métodos tradicionais: como } \\
\text { aprender Anatomia? }\end{array}$ & $\begin{array}{l}\text { 1. XXVI Congresso Brasileiro de Anatomia (CBA) e II Encontro de Ligas Estudantis } \\
\text { de Morfologia }\end{array}$ \\
\hline $\begin{array}{l}\text { Inserção de metodologias ativas no ensino da Anatomia } \\
\text { do aparelho locomotor }\end{array}$ & $\begin{array}{l}\text { 1. XXrnada de Pesquisa e Extensão da UFRJ (JPE) } \\
\text { de Morfologia }\end{array}$ \\
\hline $\begin{array}{l}\text { O método Pilates no tratamento e prevenção de } \\
\text { disfunções da coluna vertebral }\end{array}$ & $\begin{array}{l}\text { 2. Jornada de Pesquisa e Extensão da UFRJ (JPE) } \\
\text { 3. Congresso Brasileiro de Educação Médica (Cobem) }\end{array}$ \\
\hline
\end{tabular}

tomia do Rio de Janeiro, no momento composto pelas LA de Anatomia referentes à Universidade Federal do Rio de Janeiro (UFRJ), Universidade do Estado do Rio de Janeiro (Uerj), Universidade Federal do Estado do Rio de Janeiro (Unirio), Universidade Federal Fluminense (UFF), Universidade Federal Rural do Rio de Janeiro (UFRRJ), Faculdade de Medicina de Petrópolis (FMP). Este grupo discute o ensino da Anatomia e planeja o que pode ser mudado para melhor compreensão e afinidade na graduação.

Conforme relatado, a Laanamac organizou o I Simpósio de Anatomia do Campus UFRJ-Macaé, no dia 25 de agosto de 2014. A presença da presidente da Sociedade Brasileira de Anatomia, de palestrantes de outras instituições e o curso de Anatomia Palpatória foram de extrema importância para o desenvolvimento dos alunos de um campus no interior, pois proporcionaram uma experiência que não é vivida com frequência em virtude da distância dos grandes centros.

A Laanamac compõe ainda a Comissão Organizadora da primeira Jornada Interligas de Anatomia do Sudeste, um evento com a participação das LA de Anatomia de Minas Gerais, Rio de Janeiro e São Paulo. A possibilidade de participar de congressos, trocar informações com outras ligas, expor à sociedade os mais diferentes temas do cotidiano relacionados com a disciplina despertou o interesse de vários estudantes da área de saúde. Estudantes de Medicina, como acadêmicos de diversos cursos, buscam na liga a contribuição significativa que ela proporciona à aprendizagem e ao desenvolvimento do conhecimento.

\section{CONSIDERAÇÕES FINAIS}

A Laanamac trouxe ganhos para a comunidade científica e contribuiu para o crescimento regional da universidade em um campus no interior. Além disso, assim como outras ligas, funciona como instrumento de inserção na atividade médica e científica, desmistificando assuntos outrora desconhecidos ou até mesmo rejeitados ${ }^{15}$. No contexto de uma liga acadêmica recém-formada, conquistou importante espaço e proporcionou discussões na área de Anatomia. A Laanamac prioriza o conhecimento e o interesse dos participantes por essa área de atuação e proporcionou diversas experiências aos acadêmicos por meio de iniciativas de pesquisa, promoção da saúde e educação médica.

Estas experiências podem contribuir para a construção de profissionais proativos, maduros, que poderão cooperar de alguma forma com a ampliação do conhecimento da área de Anatomia e com sua introdução no contexto social, acadêmico e profissional. Acadêmicos que objetivam iniciar uma liga relevante acadêmica e socialmente precisam compreender que tudo isso foi possível no primeiro ano de existência da Laanamac, pois os objetivos foram claros e definidos desde o início, lançando propostas comprometidas com o tripé ensino-pesquisa-extensão.

\section{REFERÊNCIAS}

1. Associação Brasileira de Ligas Acadêmicas de Medicina. Diretrizes nacionais em ligas acadêmicas de medicina, São Paulo, 2011. 
2. Peres CM, Andrade AS, Garcia SB. Atividades Extracurriculares: multiplicidades de diferenciação necessárias ao curriculum. Rev. Bras. Educ. Méd. 2007;31(3):203-311.

3. Torres AR, Oliveira GM, Yamamoto FM, Lima MCP. Ligas Acadêmicas e formação médica: contribuições e desafios. Interface Comun Saúde Educ. 2008;12(27):713-720.

4. Brasil. Constituição da República federativa do Brasil. Título VIII, Cap.III, Seção I, art.207. Brasília, DF, Senado, 1988.

5. Pêgo-Fernandes PM, Mariani AW. O ensino médico além da graduação: ligas acadêmicas. Diagn Tratamento, 2011;16(2):50-51.

6. Hamamoto Filho PT, Villa-Bôas PJF, Corrêa FG, Muñoz GOC, Zaba M, Venditti VC, Schellini AS. Normatização da abertura de ligas acadêmicas: a experiência da Faculdade de Medicina de Botucatu. Rev. Bras. Educ. Méd. 2010;34(1):160-167.

7. Nogueira VMR. A importância da equipe interdisciplinar no tratamento de qualidade na área da saúde. Katálysis.1998;3:40-48.

8. Peres CM. Atividades extracurriculares: percepções e vivências durante a formação médica. Ribeirão Preto; 2006. Mestrado [Dissertação] - Faculdade de Medicina de Ribeirão Preto. Universidade de São Paulo.

9. Oliveira NA, Meirelles RMS, Cury GC, Alves LA. Mudanças curriculares no ensino médico brasileiro: um debate crucial no contexto do Promed. Rev. Bras. Educ. Méd. 2008;32(3):333-346.

10. Hamamoto Filho PT. Ligas Acadêmicas: Motivações e Críticas a Propósito de um Repensar Necessário. Rev. Bras. Educ. Med. 2011;35(4):535-543.

11. Borsatto AZ, Silva PDD, Assis F, Oliveira NCC, Rocha PR, Lopes GT. Processo de implantação e consolidação da monitoria acadêmica na UERJ. 2006;10:187-194.
12. Santos DFD, Boscaino EG, Pavão AC. Avaliação da Contribuição da Monitoria para o Desempenho do Aluno de Engenharia - Um Estudo de Caso na Escola de Engenharia Mauá. Anais do XXXIV COBENGE. 2006; p.1767-1775.

13. Ramos LAV, Costa DS, Cascaes JSA, Souza RTS, Rocha IFC, Galeno NS, Cardoso MM. Plano de monitoria acadêmica na disciplina Anatomia Humana: relato de experiência. Ensino, Saúde e Ambiente. 2012; 3(5):94-101.

14. Ramalho AS, Silva FD, Kronemberger TB, Pose RA, TSA, Carmona MJC, TSA, Auler Jr JOC, TSA. Ensino de Anestesiologia durante a Graduação por meio de uma Liga Acadêmica: qual o impacto no aprendizado dos alunos. Rev. Bras. Anestesiol. 2012;62(1):63-73.

15. Monteiro LLF. Ligas acadêmicas: o que há de positivo? Experiência de implantação da Liga Baiana de Cirurgia Plástica. Rev. Bras. Cir. Plást. 2008;23(3):158-161.

\section{CONTRIBUIÇÃO DOS AUTORES}

Os autores são fundadores da Laanamac e contribuíram para a confecção do artigo.

\section{CONFLITO DE INTERESSES}

Os autores declaram não haver conflito de interesses.

\section{ENDEREÇOS PARA CORRESPONDÊNCIA}

Jorge Henrique Santos da Silva

Av. Aluizio da Silva Gomes, 50

Granja dos Cavaleiros - Macaé

CEP 27930-560 - RJ

E-mail: jorgehenriquesantosdasilva_22@hotmail.com 